(C) 2005 American Chemical Society, J. Am. Chem. Soc., Miyauchi ja043289j Supporting Info Page 1

Supporting Information for:

\title{
Chiral Supramolecular Polymers Formed by Host-Guest
}

\section{Interactions}

Masahiko Miyauchi, Yoshinori Takashima, Hiroyasu Yamaguchi, and Akira Harada*

Department of Macromolecular Science, Graduate School of Science, Osaka University,

1-1 Machikaneyama, Toyonaka, Osaka, 560-0043, Japan

harada@chem.sci.osaka-u.ac.jp

\section{Contents}

Figure S1. ${ }^{1} \mathrm{H}$ NMR spectrum (400 MHz) of 3-p-'BocCiNH- $\alpha-\mathrm{CD}$ in DMSO- $d_{6}$.

Figure S2. ${ }^{1} \mathrm{H}$ NMR spectra ( $\left.400 \mathrm{MHz}\right)$ of 3-p- ${ }^{\mathrm{t}} \mathrm{BocCiNH}-\alpha-\mathrm{CD}$ at various concentrations in $\mathrm{D}_{2} \mathrm{O}$. Figure S3. 2D ROESY NMR spectrum ( $500 \mathrm{MHz}$ ) of 3-p- ${ }^{\mathrm{t}} \mathrm{BocCiNH}-\alpha-\mathrm{CD}$ in $\mathrm{D}_{2} \mathrm{O}$.

Figure S4. Circular dichroism spectra of 3-p-'BocCiNH- $\alpha-\mathrm{CD}$ (molar circular-dichroic absorption $(\Delta \varepsilon)$ vs. wavelength) in aqueous solutions (a) and plots of the intensities $(\Delta \varepsilon)$ of the observed Cotton bands around 327 and $288 \mathrm{~nm}$ as a function of concentrations (b).

Figure S5. Circular dichroism spectra of $3-p-{ }^{t} \mathrm{BocCiNH}-\alpha-\mathrm{CD}(1.5 \mathrm{mM})$ at various temperatures in water.

Figure S6. Stejskal-Tanner plots of 3-p-'BocCiNH- $\alpha-\mathrm{CD}$ at $5.0 \mathrm{mM}(\boldsymbol{\bullet}), 10 \mathrm{mM}(\mathbf{\Delta}), 20 \mathrm{mM}(\downarrow)$ and $40 \mathrm{mM}(\bullet)$.

Figure S7. Plots of the diffusion coefficients (D) of 3-p-'BocCiNH- $\alpha-\mathrm{CD}(0)$ and TMA (a) vs. concentration.

Table S1. Diffusion coefficients (D) of $3-p-{ }^{t} \mathrm{~B} O \mathrm{CC} \mathrm{NNH}-\alpha-\mathrm{CD}$ and TMA, and ratio of diffusion coefficients ( $r$ ) of 3- $p-{ }^{\prime} \mathrm{BocCiNH}-\alpha-\mathrm{CD}$ for TMA.

Table S2. Relative viscosity $\left(\eta_{r}\right)$, specific viscosity $\left(\eta_{s p}\right)$ and reduced viscosity $\left(\eta_{\text {sp }} /\right.$ Conc.) of $3-p-{ }^{t} \mathrm{BocCiNH}-\alpha-\mathrm{CD}$ in water. 
(C) 2005 American Chemical Society, J. Am. Chem. Soc., Miyauchi ja043289j Supporting Info Page 2

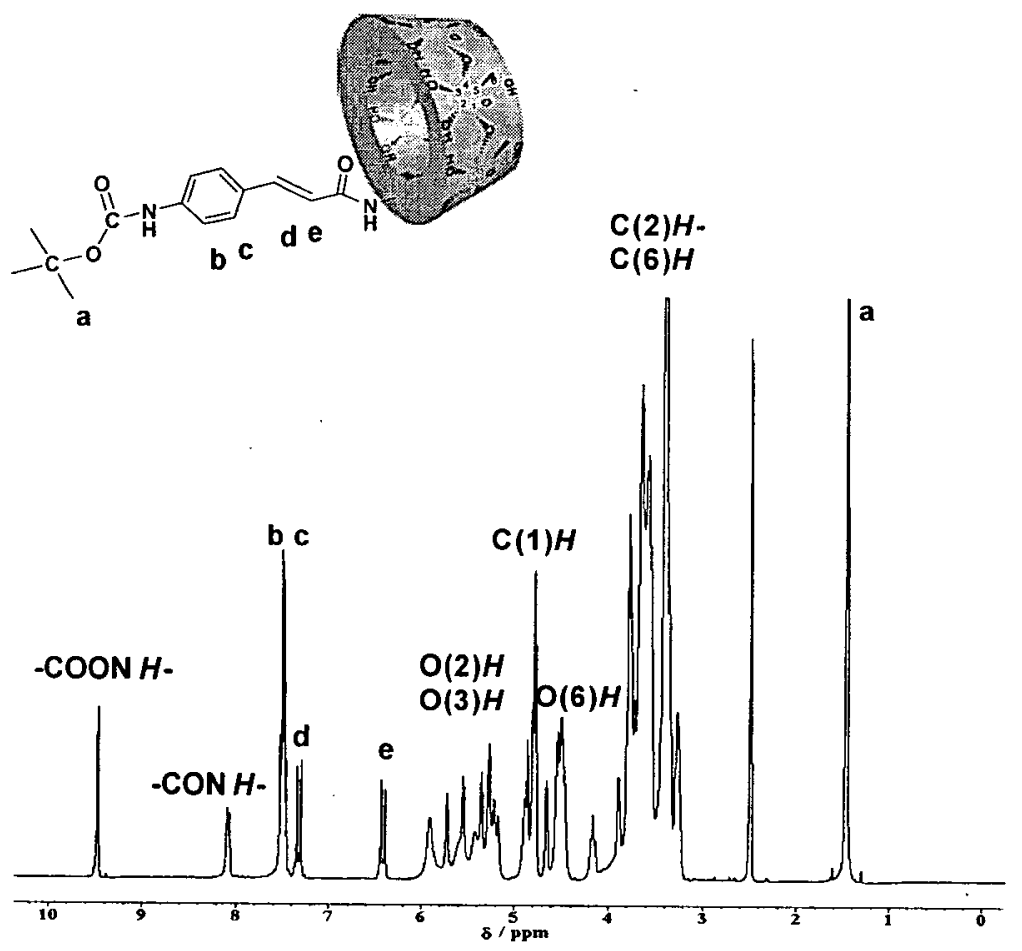

Figure S1. ${ }^{1} \mathrm{H}$ NMR spectrum (400 MHz) of $3-p-{ }^{t} \mathrm{BocCiNH}-\alpha-\mathrm{CD}$ in DMSO- $d_{6}$. 
(C) 2005 American Chemical Society, J. Am. Chem. Soc., Miyauchi ja043289j Supporting Info Page 3

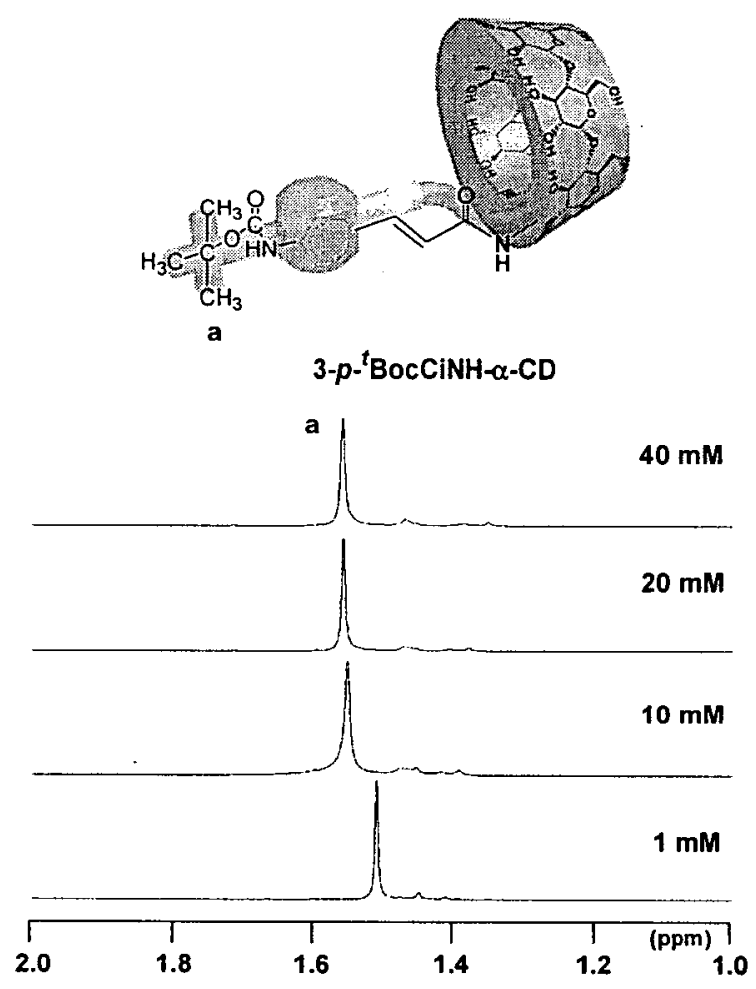

Figure S2. ${ }^{1} \mathrm{H}$ NMR spectra $(400 \mathrm{MHz})$ of $3-p-{ }^{t} \mathrm{BocCiNH}-\alpha-\mathrm{CD}$ at various concentrations in $\mathrm{D}_{2} \mathrm{O}$. 
(C) 2005 American Chemical Society, J. Am. Chem. Soc., Miyauchi ja043289j Supporting Info Page 4
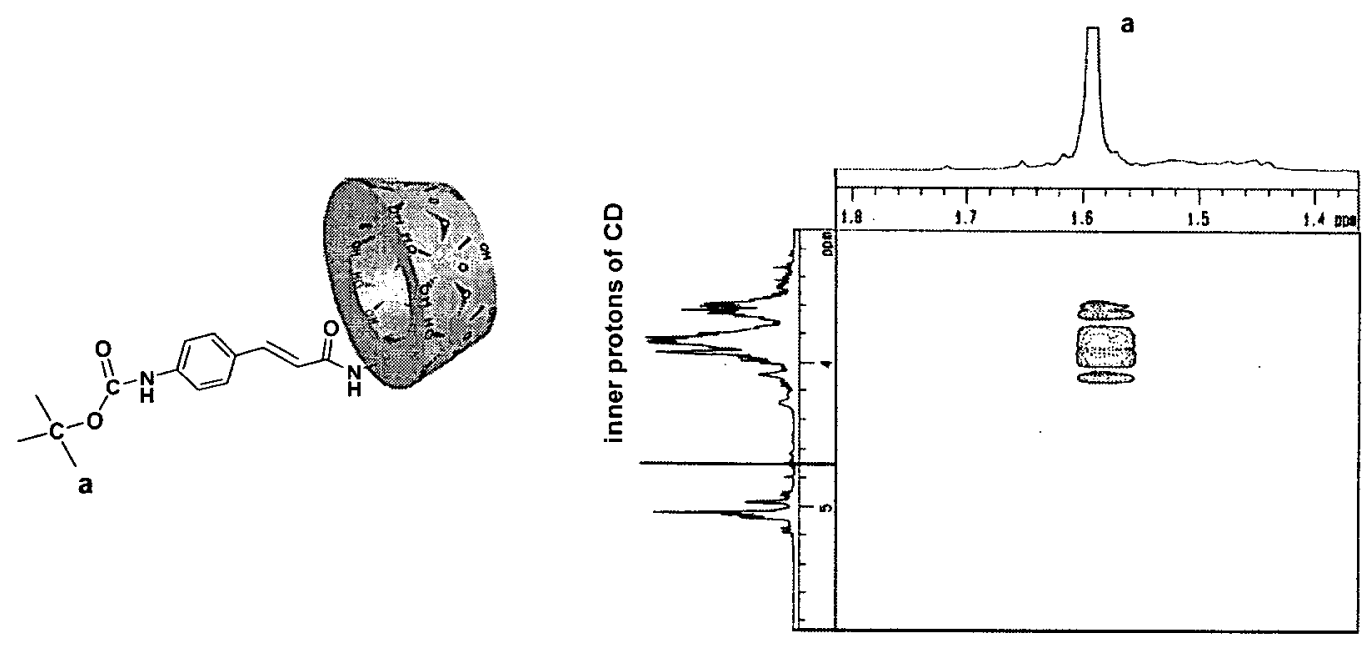

Figure S3. 2D ROESY NMR spectrum (500 MHz) of 3-p-'BocCiNH- $\alpha-C D$ in $\mathrm{D}_{2} \mathrm{O}$ 
(C) 2005 American Chemical Society, J. Am. Chem. Soc., Miyauchi ja043289j Supporting Info Page 5

a)

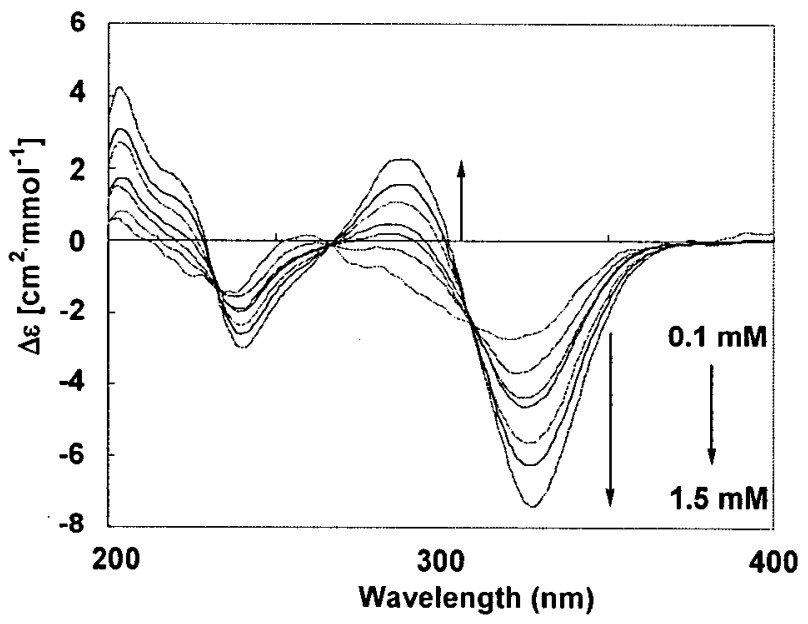

(b)

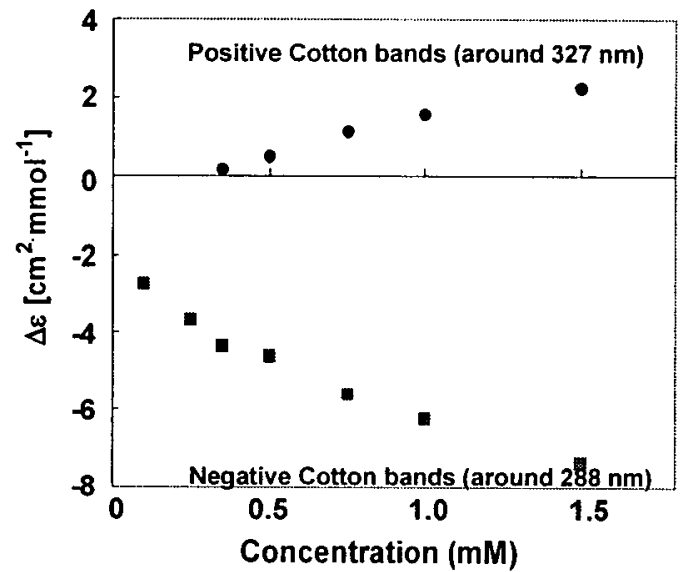

Figure S4. Circular dichroism spectra of 3-p- ${ }^{t} \mathrm{BocCiNH}-\alpha-\mathrm{CD}$ (molar circular-dichroic absorption $(\Delta \varepsilon)$ vs. wavelength) in aqueous solutions (a) and plots of the intensities $(\Delta \varepsilon)$ of the observed Cotton bands around 327 and $288 \mathrm{~nm}$ as a function of concentrations (b). 
(C) 2005 American Chemical Society, J. Am. Chem. Soc., Miyauchi ja043289j Supporting Info Page 6

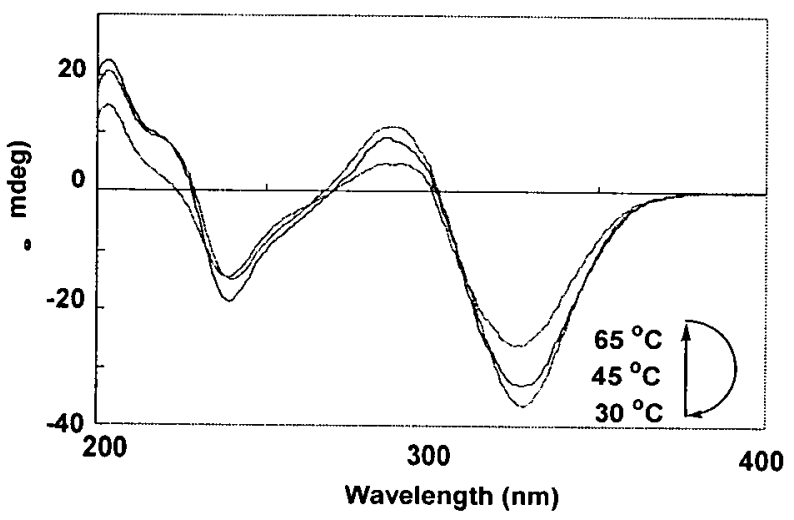

Figure S5. Circular dichroism spectra of $3-p-{ }^{t} \mathrm{BocCiNH}-\alpha-\mathrm{CD}(1.5 \mathrm{mM})$ at various temperatures in water. 
(C) 2005 American Chemical Society, J. Am. Chem. Soc., Miyauchi ja043289j Supporting Info Page 7

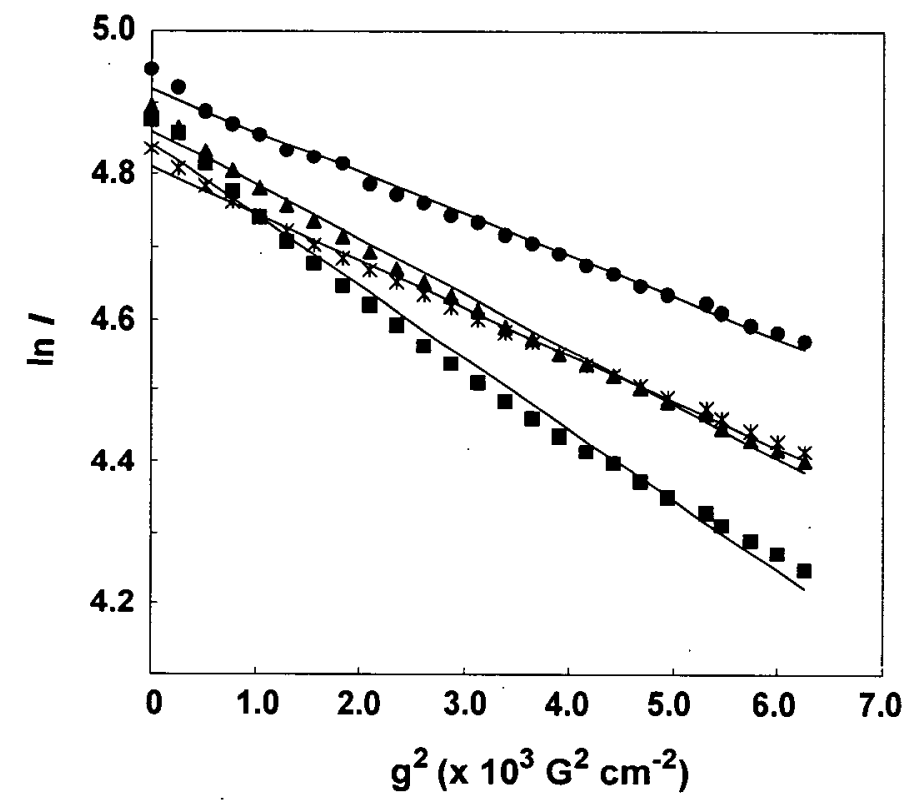

Figure S6. Stejskal-Tanner plots of 3-p-'BocCiNH- $\alpha-\mathrm{CD}$ at $5.0 \mathrm{mM}(\mathbf{-}), 10 \mathrm{mM}(\mathbf{\Delta}), 20 \mathrm{mM}(*)$ and $40 \mathrm{mM}(\bullet)$. 
(C) 2005 American Chemical Society, J. Am. Chem. Soc., Miyauchi ja043289j Supporting Info Page 8

Table S1. Diffusion coefficients $\left(\times 10^{6} \mathrm{~cm}^{2} \cdot \mathrm{s}^{-1}\right)$ of $3-p-{ }^{t} \mathrm{BocCiNH}-\alpha-\mathrm{CD}$ and TMA, and ratio of

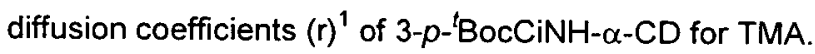

\begin{tabular}{ccccc} 
Conc. $(\mathrm{mM})$ & 5.0 & 10.0 & 20.0 & 40.0 \\
\hline \hline $\mathrm{D}_{\alpha}$ & 2.65 & 2.17 & 1.78 & 1.52 \\
$\mathrm{D}_{\text {TMA }}$ & 7.69 & 7.52 & 7.16 & 6.88 \\
$\mathrm{r}$ & 0.345 & 0.289 & 0.248 & 0.22
\end{tabular}

1) $\mathbf{r}=\left(\mathrm{D}_{\alpha} / \mathrm{D}_{\mathrm{TMA}}\right)$

2) $D_{\alpha}$ are diffusion coefficients of 3-p-BocCiNH- $\alpha-C D$.

3) $D_{T M A}$ are diffusion coefficients of TMA. 
(C) 2005 American Chemical Society, J. Am. Chem. Soc., Miyauchi ja043289j Supporting Info Page 9

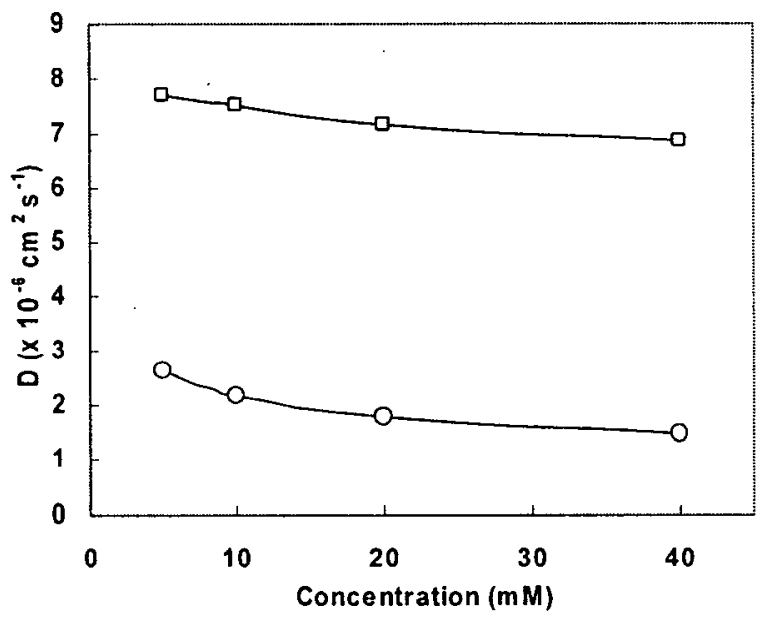

Figure S7. Plots of the diffusion coefficients (D) of $3-p-{ }^{t} \mathrm{BocCiNH}-\alpha-\mathrm{CD}(0)$ and TMA (a) vs. concentration. 
(C) 2005 American Chemical Society, J. Am. Chem. Soc., Miyauchi ja043289j Supporting Info Page 10

Table S2. Relative Viscosity $\left(\eta_{r}\right)$, Specific Viscosity $\left(\eta_{\mathrm{sp}}\right)$ and Reduced Viscosity $\left(\eta_{\mathrm{sp}} /\right.$ Conc.) of 3-p- ${ }^{t} \mathrm{BocCiNH}-\alpha-\mathrm{CD}$ in Water.

\begin{tabular}{|c|c|c|c|}
\hline Conc. $(\mathrm{g} / \mathrm{dL})$ & $\eta_{\mathrm{r}}$ & $\eta_{\text {sp }}$ & $\eta_{\text {sp }} /$ Conc. $\left(\mathrm{cm}^{3} \cdot \mathrm{g}^{-1}\right)$ \\
\hline \hline $0.61(5 \mathrm{mM})$ & 1.017 & 0.017 & 2.723 \\
\hline $1.22(10 \mathrm{mM})$ & 1.036 & 0.036 & 2.952 \\
\hline $2.43(20 \mathrm{mM})$ & 1.085 & 0.085 & 3.514 \\
\hline $4.87(40 \mathrm{mM})$ & 1.191 & 0.191 & 3.929 \\
\hline $9.74(80 \mathrm{mM})$ & 1.460 & 0.460 & 4.723 \\
\hline
\end{tabular}

